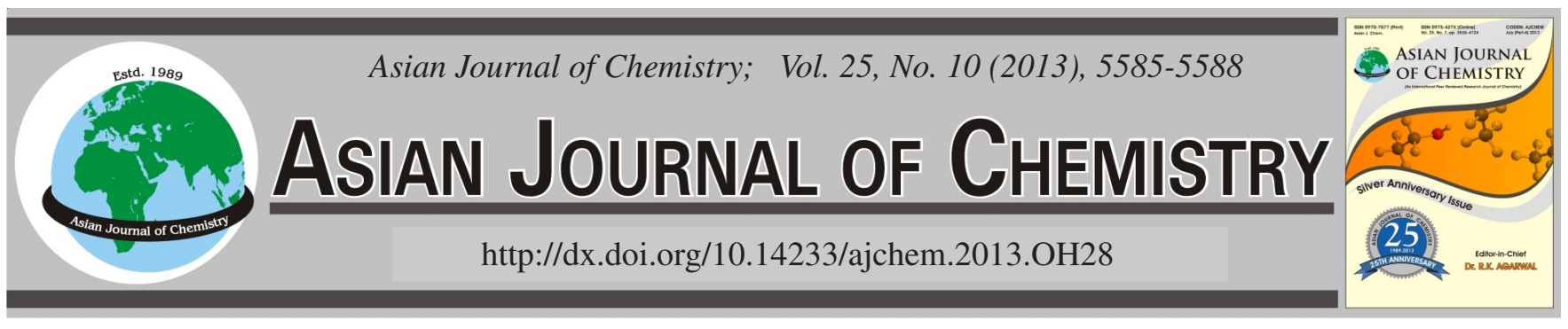

\title{
Synthesis and Characterization of St-AN-(KH-570) Block Macromolecular Coupling Agent Copolymers $\dagger$
}

\author{
Guojun Cheng ${ }^{1,2}$, Ruijuan Song ${ }^{1}$, Jibing MiaO ${ }^{1}$ and Jiasheng Qian ${ }^{1, *}$
}

${ }^{1}$ College of Chemistry and Chemical Engineering, Anhui University, Hefei 230039, P.R. China

${ }^{2}$ School of Materials Science and Engineering, Anhui University of Science and Technology, Huainan 232001, P.R. China

*Corresponding author: Tel: +86 551 5107342; E-mail: qianjsh@ahu.edu.cn

\begin{abstract}
A new well-defined amphiphilic St-AN-(KH-570) block macromolecular coupling agent copolymers was successfully synthesized via free radical polymerization with styrene (St), acrylonitrile (AN) and $\gamma$-methacryloxypropyl trimethoxy silane (KH-570) in dimethylbenzene. The compositions of copolymers were confirmed by Fourier transform infrared spectroscopy and proton nuclear magnetic resonance. Thermal analysis by differential scanning calorimetry indicated that glass transition temperature $\left(\mathrm{T}_{\mathrm{g}}\right)$ of macromolecular coupling agent is much lower than polystyrene and polyacrylonitrile. The synthesis possessed the number average molecular weights $\left(\mathrm{M}_{\mathrm{n}}\right)$ of $1.05 \times 10^{4}$ by gel permeation chromatography with broad polydispersity index value of 2.48 .
\end{abstract}

Key Words: Amphiphilic copolymer, Macromolecular coupling agent, $\gamma$-Methacryloxypropyl trimethoxy silane.

ᄂ - - - - - - - - - - - - - - - - - - - - - - - - - - - -

\section{INTRODUCTION}

The synthesis of well-defined amphiphilic functionalized macromolecular coupling agent has attracted a great deal of interest due to its improved properties in adhesion, compatibility with other polymer materials ${ }^{1-5}$. Many techniques have been used to synthesize the amphiphilic functionalized block copolymers, one of the most important techniques is atom transfer radical polymerization, because this method has been developed to obtain narrow polydispersity index, architectures, functionalities and well-defined compositions ${ }^{6-8}$. However, there are a few limitations for normal atom transfer radical polymerization. For example, its catalyst is sensitive to air and the catalyst concentration is independently reduced ${ }^{9,10}$. They impede the use of atom transfer radical polymerization in the commercial production of functionalized macromolecular coupling agent copolymers. In order to overcome the drawbacks of application of commercial production,we directly utilize free radical polymerization with monomers to prepare amphiphilic functionalized block copolymers. The method not only can increase production also can widen its application in polymer blends, thermoplastic elastomers and polymer nanocomposites ${ }^{3}$.

In previous work, coupling agent $\mathrm{KH}-570$ was applied to modify nanoparticles and prepare nanoparticles/rubber composites. Due to the strong interface action, this nanocomposite exhibit unique hybrid properties. including good heat-resistance, good wear-resistance and good dynamic mechanical properties. In order to further study the effect of molecule weight to polymer. St-AN-(KH-570) block macromolecular coupling agent copolymers was synthesized via free radical polymerization with styrene, acrylonitrile and $\mathrm{KH}-570$ as material monomers. The polymerization course of block copolymer is shown in Fig. 1.

In the study, a new block macromolecular coupling agent [St-AN-(KH-570)] was designed and synthesized in dimethylbenzene system using benzoyl peroxide as initiator<smiles>CO[Si](CCOC(=O)C(C)=CC[C@@H](C#N)C=CC=Cc1ccccc1)(OC)OC</smiles><smiles>CO[Si](C)(OC)OCC(=O)C(C)C(C)C=CCC(C)c1ccccc1</smiles>

Fig. 1. Synthesis of St-AN-(KH-570) block copolymer was carried out according to the scheme

$†$ Presented to the 6th China-Korea International Conference on Multi-functional Materials and Application, 22-24 November 2012, Daejeon, Korea 
and dodecyl thioalcohol (DDM) as chain transfer agent.On the one hand, $\mathrm{KH}-570$ as the side group $\left[\mathrm{Si}-\mathrm{OCH}_{3}\right]$ on the main chain may react with - $\mathrm{OH}$ and -NH which are on the surface of nanoparticles containing active groups. On the other hand, St-AN-(KH-570) copolymer has the quite similar structure or polarity with rubbers and provides them great compatibility.

\section{EXPERIMENTAL}

Styrene (St), acrylonitrile (AN) and dimethylbenzene (Shanghai Chemical Solvent Factory) were purified by distillation at reduced pressure before use. $\gamma$-Methacryloxypropyl trimethoxy silane (KH-570) was purchased from Nanjing Shuguang Chemical Group Co. Ltd., in China. Benzoyl peroxide (BPO) (Shanghai Chemical Solvent Factory) was purified by recrystallization from chloroform. Dodecyl thioalcohol was pruchased from Shanghai Chemical Solvent Factory and was used without further purification. Other reagents were of analytical grade and used as received.

Synthesis and purification of St-AN-(KH-570) block copolymers: $100 \mathrm{~mL}$ of dimethylbenzene was used for the solvent, was added into a $250 \mathrm{~mL}$ three necked roundbottomed flask with constantly stirring and bubbling of a slow stream of nitrogen gas for $c a .0 .5 \mathrm{~h}$ at $80^{\circ} \mathrm{C}$ in a temperaturecontrolled water-bath. $10 \mathrm{~g}$ of St, $5 \mathrm{~g}$ of acrylonitrile, $5 \mathrm{~g}$ of $\mathrm{KH}-570,0.5 \mathrm{~g}$ of dodecyl thioalcohol (DDM) and $0.5 \mathrm{~g}$ of benzoyl peroxide dissolved in $30 \mathrm{~mL}$ dimethylbenzene, which were mixed and slowly added into the flask to initiate the block copolymer. After $3 \mathrm{~h}$, the reaction was stopped by letting air into the reactor and cooling the flask. The product was precipitated by pouring the reaction mixture into methanol. The precipitate was filtered, washed thoroughly with methanol, then vacuum drying at $45^{\circ} \mathrm{C}$ for $24 \mathrm{~h}$.

Instrumental analysis: The compositions of St-AN-(KH570) block copolymers were investigated by FTIR (Nicolet Co., Neuxs-830, USA) with a resolution of $4 \mathrm{~cm}^{-1}$ for which the samples were palletized with $\mathrm{KBr}$ powder. ${ }^{1} \mathrm{H} \mathrm{NMR}$ spectra were performed by a Bruker Avance 400 spectrometer (Switzerland) and the deuterated chloroform-d $\left(\mathrm{CDCl}_{3}\right)$ was usded for solvent to obtain the spectra. Molecular weights and polydispersities of product were determined by GPC equipment (Varian Co., USA), which was used for data collecting and processing. $100 \mu \mathrm{L}$ of samples were injected as a solution in tetrahydrofuran and tetrahydrofuran was used as a mobile phase at flow rate of $0.8 \mathrm{~mL} \mathrm{~min}^{-1}$. The calibration of the photometer was carried out by normalization with a polystyrene standard. Differential scanning calorimetry was performed using Q2000 apparatus (TA Co., USA) for a temperature range from $0{ }^{\circ} \mathrm{C}$ to $120{ }^{\circ} \mathrm{C}$, at a heating rate of $10{ }^{\circ} \mathrm{C} / \mathrm{min}$ under nitrogen atmosphere.

\section{RESULTS AND DISCUSSION}

FTIR analysis: Fig. 2. shows the FTIR spectra of monomers and block copolymer. The characteristic adsorption peaks of $\mathrm{KH}-570$, carboxyl $\mathrm{C}=\mathrm{O}$ at $1730 \mathrm{~cm}^{-1}, \mathrm{Si}-\mathrm{OH}$ at 1300 $\mathrm{cm}^{-1}$, Si-O-C at $1080 \mathrm{~cm}^{-1}$ and $-\mathrm{CH}_{3}$ at region $3000-2800 \mathrm{~cm}^{-1}$, were observed in the spectrum of St-AN-(KH-570) block copolymer. nitrile group - $\mathrm{CN}$ at $2240 \mathrm{~cm}^{-1}$ was found in spectrum of copolymer. Meanwhile, the absorption peaks at $3080 \mathrm{~cm}^{-1}$, in the region $2000-1700 \mathrm{~cm}^{-1}$ and $1600-1450 \mathrm{~cm}^{-1}$ correspond to the Ar-H (stretching vibration mode), =C-H (out-of-plane bending vibration mode of benzene ring) and skeleton vibrations of benzene ring, respectively ${ }^{11-13}$. These characteristic adsorption peaks of monomers were found in the spectrum of block copolymers as well. But the $-\mathrm{C}=\mathrm{C}$ group $\left(1640 \mathrm{~cm}^{-1}\right)$ of monomers was not observed in spectrum of St-AN-(KH-570) block copolymer. Therefore, FTIR spectra of copolymer indicates block macromolecular coupling agent copolymers have been successfully synthesized via free radical polymerization.

${ }^{1}$ H NMR Analysis: Fig. 3 shows the ${ }^{1} \mathrm{H}$ NMR spectrum of the prepared St-AN-(KH-570) block copolymer. The spectrum shows the strong adsorption peaks at $0.8-0.9,1.2,1.5,2.0$, 2.2-2.4, 3.4-3.5, 6.5-6.7 and 7.05-7.07 ppm. The adsorption peaks at 7.05-7.07, 2.2-2.4 and 0.8-0.9 ppm are assigned to be the polystyrene segment, those at 6.5-6.7 and weak adsorption peaks at 5.3-5.4 ppm are attributed to polyacrylonitrile segment. Meanwhile those at 0.8-0.9, 1.2, 1.5, 2.0 and 3.4-3.5 ppm are attributed to poly $(\mathrm{KH}-570)^{14,15}$. These chemical shifts show the successful synthesis of the prepared St-AN-(KH570) block macromolecular coupling agent copolymer.

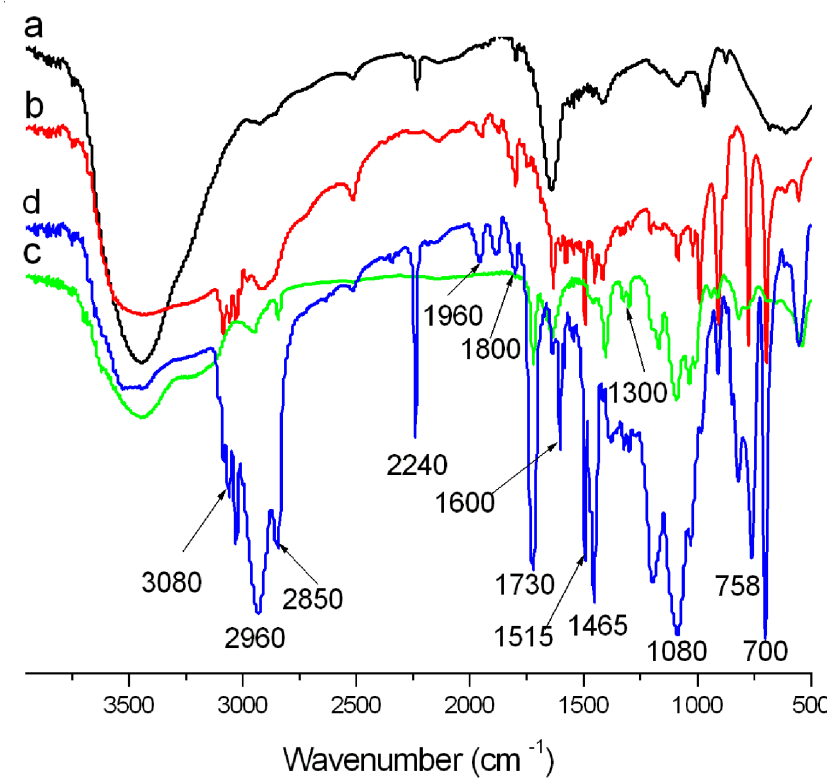

Fig. 2. FTIR spectra of (a) acrylonitrile; (b) styrene; (c) KH-570; (d) StAN-(KH-570) copolymer

GPC anlysis: Fig. 4 shows the GPC curves of the prepared amphiphilic St-AN-(KH-570) block copolymer. The copolymer shows unimodal molecular weight distribution in the GPC curves, indicating that no or negligible homopolymer residue in the copolymer products. The result of GPC shows that the polydispersity index of amphiphilic St-AN-(KH-570) block copolymer is broader than 1.0. The average molecular weight (Mn) and polydispersity index of the prepared St-AN-(KH-570) block copolymer is listed in Table-1. The molecular weight of amphiphilic St-AN-(KH-570) block copolymer conforms to the design requirements of macromolecular coupling agent.

Differential scanning calorimetry analysis: Thermal analysis performed by differential scanning calorimetry as shown in Fig. 5 did not detect melting peak indicating fully amorphous morphology of St-AN-(KH-570) block copolymer. 


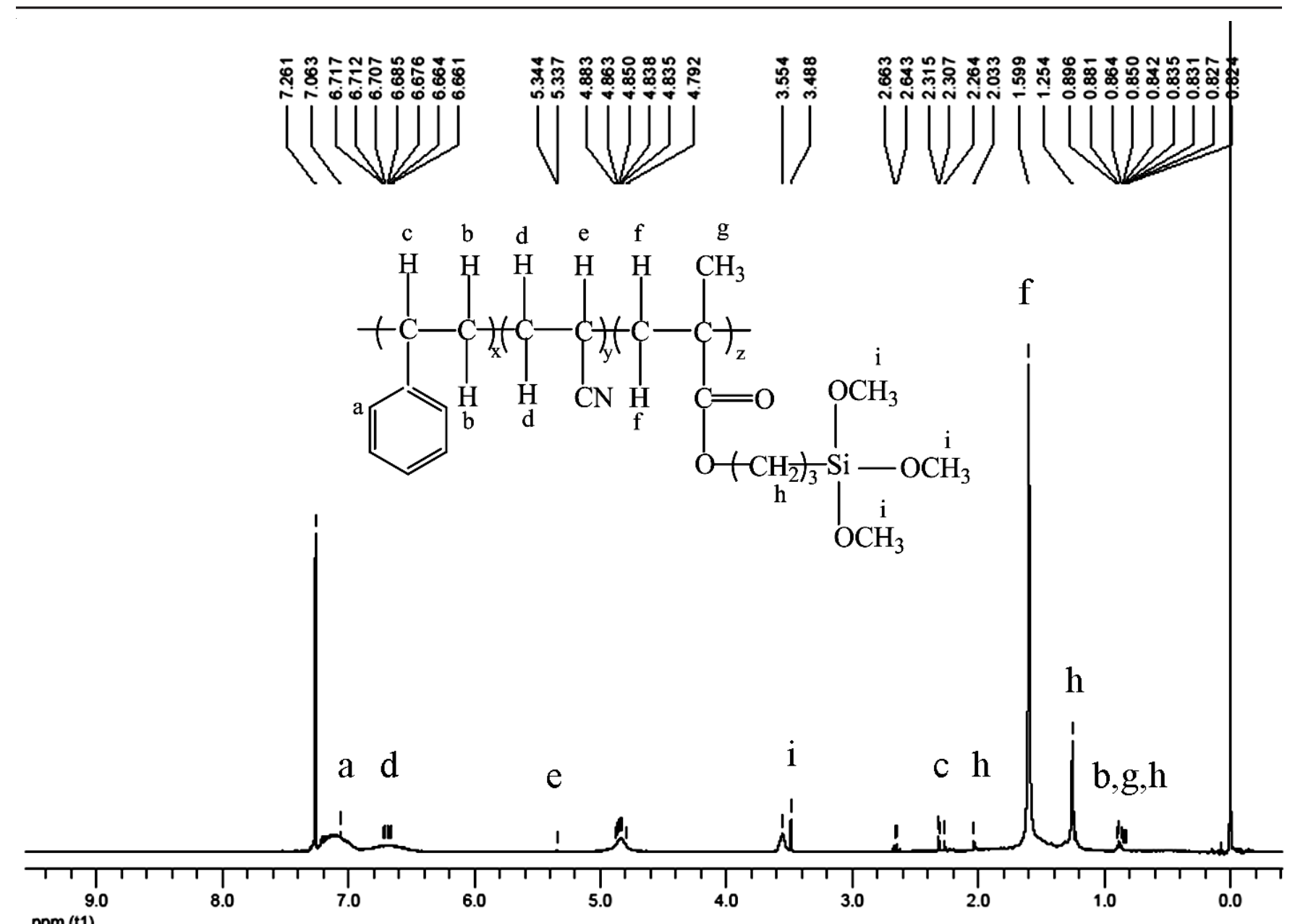

Fig. 3. ${ }^{1} \mathrm{H}$ NMR spectrum of prepared St-AN-(KH-570) block copolymer

In contrast to the glass transition temperature $\left(\mathrm{T}_{\mathrm{g}}\right)$ of PS and $\mathrm{PAN}^{16}, \mathrm{~T}_{\mathrm{g}}$ determined at first heating run (Fig. 5) appeared as single step transition on differential scanning calorimetry curve presenting no phase separation. It can be observed from Fig. 5 that block copolymer's $\mathrm{T}_{\mathrm{g}}\left(55.5^{\circ} \mathrm{C}\right)$ is much lower than PS $\left(124{ }^{\circ} \mathrm{C}\right)$ and PAN $\left(80-100{ }^{\circ} \mathrm{C}\right)$. Therefore, St-AN-(KH-570) block macromolecule coupling agent copolymer is stable products and can be useful for longer time in the well-soluble form.

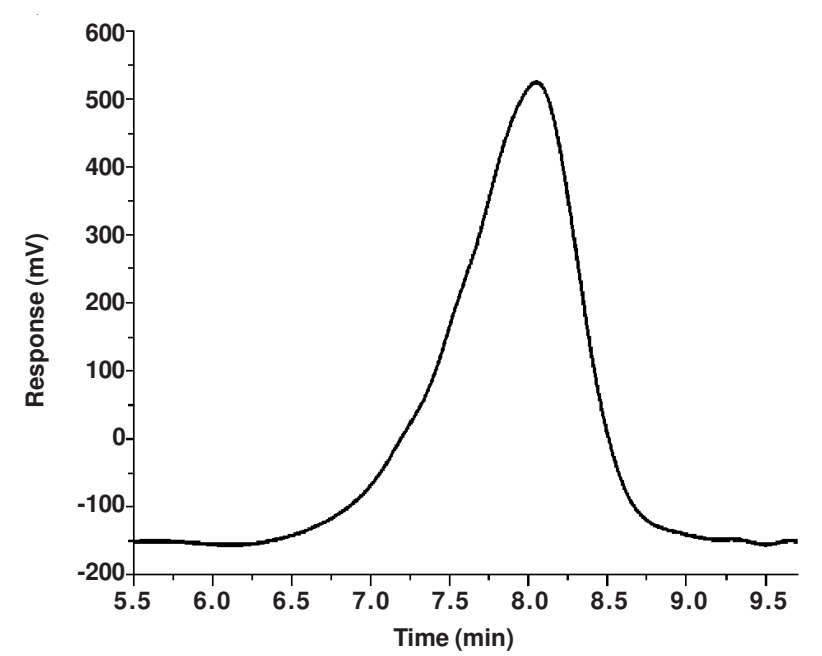

Fig. 4. GPC curves of block copolymer
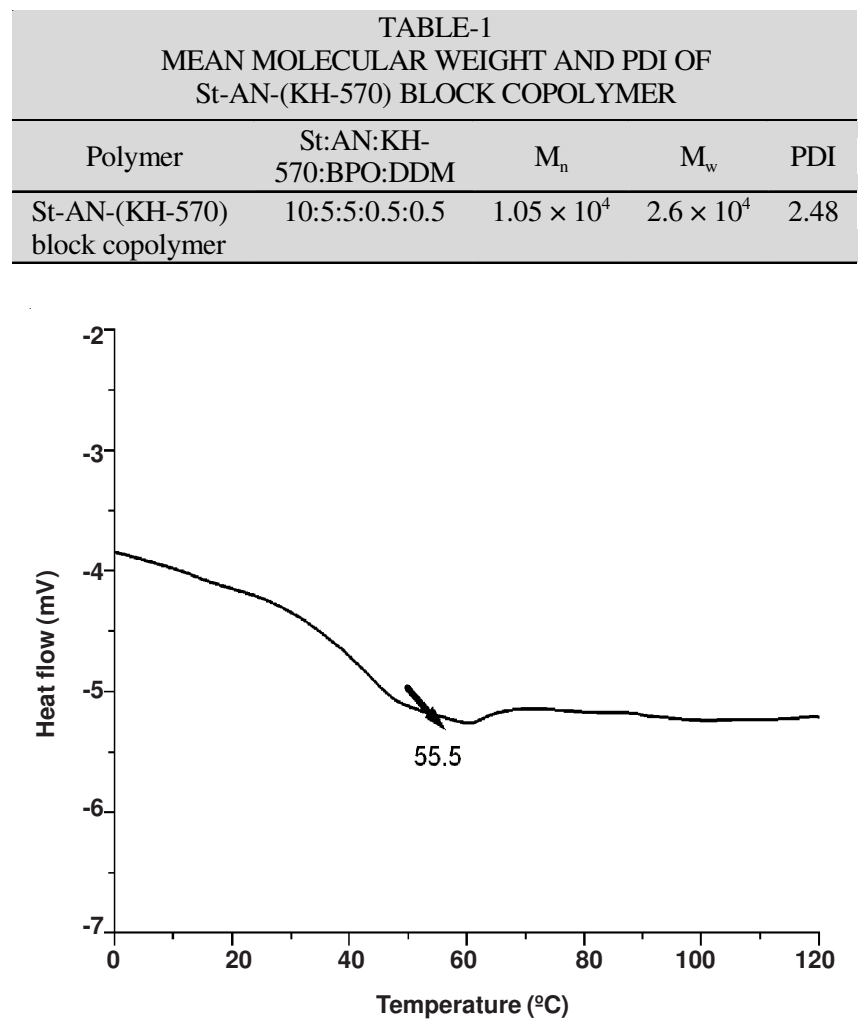

Fig. 5. Differential scanning calorimetry curve of block copolymer 


\section{Conclusion}

A well-defined amphiphilic block macromolecular coupling agent copolymer (St-AN-(KH-570)) was highly effectively synthesized via free radical polymerization with styrene, acrylonitrile and KH-570 in dimethylbenzene. The GPC results showed that the polydispersity index of St-AN(KH-570) copolymer was broader than 1.0 and conformed to the design requirements of macromolecular coupling agent. The analysis of FTIR and ${ }^{1} \mathrm{H}$ NMR confirmed the structure of the prepared St-AN-(KH-570) copolymer. Differential scanning calorimetry curve showed that block copolymer's $T_{g}$ is much lower than polystyrene and polyacrylonitrile.

\section{ACKNOWLEDGEMENTS}

This research was supported by the Research Fund for the Doctoral Program of Higher Education of China (20113401110003), the National Natural Science Foundation of China (51273001/E030703) and "211 Project" of Anhui University.

\section{REFERENCES}

1. J. Feng, H. Liu and Y. Huan, Fluid Phase Equilib., 261, 50 (2007).

2. D. Gromadzki, J. Lokaj, M. Slouf and P. Stepanek, Polymer, 50, 2451 (2009).

3. Y.L. Tai, J.S. Qian and Y.C. Zhang, Chem. Eng. J., 141, 354 (2008).

4. Y. Sun and W.Q. Liu, J. Fluorine Chem., 132, 9 (2011).

5. R.S. Plentz, M. Miotto and E.E. Schneider, J. Appl. Polym. Sci., 101, 1799 (2006).

6. W.L. Qiu, K.C. Mai and H.M.Zeng, J. Appl. Polym. Sci., 71, 1537 (1999).

7. L.Y. Wang, K.C. Li and H.C. Lin, Polymer, 51, 75 (2010).

8. R. Liu, Z.Y. Li, D. Yuan and C.F. Meng, Polymer, 52, 356 (2011).

9. R. Xia, Y.C. Zhang and Q.R. Zhu, J. Appl. Polym. Sci., 107, 562 (2008).

10. R. Xia, M.H. Li, Y.C. Zhang, Q.R. Zhu and J.S. Qian, J. Appl. Polym. Sci., 107, 1100 (2008).

11. H.C. Dong, P.L. Ye, M.J. Zhong and J. Pietrasik, Langmuir, 19, 15567 (2010).

12. Y.Y. Yu, W.C. Chien, Y.-H. Ko, Y.-C. Chan and S.-C. Liao, Curr. Appl. Phys., 12, S7 (2012).

13. Y.J. Xie, C.A.S. Hill, Z.F. Xiao and H. Militz, Composites: Part A, 41, 806 (2010).

14. Z. Hu, X. Shen, H. Qiu and G. Lai, Eur. Polym. J., 45, 2313 (2009).

15. H. Chen, Y. Liang, D. Liu, Z. Tan and S. Zhang, Mater. Sci. Eng. C, 30, 605 (2010).

16. K. Bury, D. Neugebauer and T. Biela, React. Funct. Polym., 71, 616(2011). 